\title{
Indicadores de bem-estar subjetivo e saúde mental em mulheres de diferentes orientações sexuais
}

\author{
Fernanda de Oliveira Paveltchuk ${ }^{1}$ \\ Juliane Callegaro Borsa ${ }^{2}$ \\ Bruno Figueiredo Damásio ${ }^{3}$ \\ ${ }^{1}$ Pontifícia Universidade Católica do Rio de Janeiro, RJ, Brasil \\ https://orcid.org/0000-0002-8319-874X \\ ${ }^{2}$ Pontifícia Universidade Católica do Rio de Janeiro, RJ, Brasil \\ https://orcid.org/0000-0001-7703-5509 \\ ${ }^{3}$ Universidade Federal do Rio de Janeiro, RJ, Brasil \\ https://orcid.org/0000-0002-1150-092X
}

\begin{abstract}
Resumo
Mulheres lésbicas e bissexuais apresentam altos índices de psicopatologias, como depressão e ansiedade. Este estudo buscou comparar os índices de felicidade subjetiva, satisfação com a vida, depressão, ansiedade e estresse em mulheres lésbicas, bissexuais e heterossexuais. Participaram desse estudo 736 mulheres (heterossexuais $=414$; lésbicas $=143$; bissexuais $=194)$, maiores de 18 anos $(M=27,37 ; D P=13,44)$. As participantes responderam a um questionário online composto por questionário sociodemográfico e escalas para avaliação das variáveis psicológicas citadas. Lésbicas e bissexuais apresentaram menores níveis de bem-estar e maiores de psicopatologias do que as heterossexuais. Bissexuais apresentaram menores índices de bem-estar e maiores de psicopatologias comparadas a lésbicas e a heterossexuais. Acredita-se que esses indicadores podem ser explicados pela condição de vulnerabilidade psicossocial a qual as mulheres não heterossexuais se encontram. Pesquisas mais amplas devem ser conduzidas para a obtenção maior compreensão das variáveis preditoras dos desfechos de saúde mental nesse grupo.
\end{abstract}

Palavras-chave: lésbicas, mulheres bissexuais, bem-estar subjetivo, depressão, ansiedade.

\section{Subjective well being and mental health of women of diverse sexual orientations}

\begin{abstract}
Lesbian and bisexual women present high rates of psychopathologies, such as depression and anxiety. This study aimed to compare the subjective happiness, life satisfaction, depression, anxiety, and stress levels in lesbian, bisexual and heterosexual women. Participated in this study 736 women (heterosexuals $=414$, lesbian $=143$, bisexuals $=194)$, older than 18 years $(M=27.37, S D=13.44)$. Participants answered an online questionnaire composed of a sociodemographic questionnaire and scales for the evaluation of the mentioned psychological variables. Lesbians and bisexuals had lower levels of well-being and greater psychopathologies than heterosexuals. Bisexuals had lower rates of well-being and higher rates of psychopathology when compared to lesbians and heterosexuals. It is believed that these indicators can be explained by the condition of psychosocial vulnerability to which non-heterosexual women meet. Larger research should be conducted to gain a better understanding of the predictors of mental health outcomes in this group.
\end{abstract}

Keywords: lesbians, bisexual women, subjective well-being, depression; anxiety. 


\title{
Bienestar subjetivo y salud mental de mujeres de diferentes orientaciones sexuales
}

\begin{abstract}
Resumen
Mujeres lesbianas y bisexuales presentan altos índices de psicopatologías. Este estudio comparó los índices de felicidad subjetiva, satisfacción con la vida, depresión, ansiedad y estrés en mujeres lesbianas, bisexuales y heterosexuales. Las participantes fueran 736 mujeres (heterosexuales $=414$, lesbianas $=143$, bisexuales $=194)$, mayores de 18 años $(M=27,37, D P=13,44)$. Fue respondido un cuestionario online compuesto por cuestionario sociodemográfico y escalas para la evaluación de las variables psicológicas citadas. Lesbianas y bisexuales presentaron menores niveles de bienestar y mayores de psicopatologías que heterosexuales. Bisexuales presentaron menores índices de bienestar y mayores de psicopatologías cuando comparadas a lesbianas y heterosexuales. Se cree que estos indicadores pueden ser explicados por la condición de vulnerabilidad psicosocial a la que se encuentran las mujeres no heterosexuales. Investigaciones más amplias deben ser conducidas para obtener mayor comprensión de las variables predictoras de los resultados de salud mental en ese grupo.
\end{abstract}

Palabras clave: lesbianas, mujeres bisexuales, bienestar subjetivo, depresión, ansiedad.

Em geral, a heterossexualidade é culturalmente entendida como a única forma aceitável de sexualidade e, por isso, a homossexualidade é considerada como um modo desviante de orientação de afetos e atrações. Tais concepções podem provocar comportamentos violentos e hostis contra lésbicas, gays e bissexuais (Borrillo, 2010). A isso se dá o nome de homofobia (Borrillo, 2010). A homofobia pode ser internalizada ou externa (Dunn et al., 2013). A do tipo internalizada, diz respeito às atitudes negativas que alguém que se identifique como gay, lésbica ou bissexual desenvolve a respeito da homossexualidade ou bissexualidade ao longo de sua história de vida. Ou seja, é uma homofobia autodirigida. A homofobia externalizada, por sua vez, está associada a atos de preconceito e discriminação, sejam eles verbais ou físicos, contra indivíduos LGB (Mustanski, Andrews, \& Puckett, 2016). Deste modo, trata-se de uma homofobia heterodirigida. A homofobia, tanto internalizada quanto externalizada, produz eventos que podem ser considerados estressores pelos indivíduos LGB, podendo aumentar as suas inclinações a desenvolver depressão, ansiedade e estresse (Meyer, 2015). O medo de rejeição de amigos e familiares devido à homo ou à bissexualidade revelada é um fator de estresse para os indivíduos LGB que ainda têm suas orientações sexuais ocultadas de seus pares (Mayock, Bryan, Carr, \& Kitching, 2008).
A discriminação de gênero pode impactar negativamente o bem-estar de mulheres, devido à posição de desvantagem em que são tidas, quando comparadas aos homens, na sociedade (Schmitt, Branscombe, Kobrynowicz, \& Owen, 2002). Quando comparadas às mulheres heterossexuais, mulheres lésbicas e bissexuais apresentam altos índices de psicopatologias como depressão e ansiedade, assim como grande propensão a comportamentos de risco como abuso de substâncias, autodano e suicídio (Donahue et al., 2017). Não existem evidências de que isso se deva à sua orientação sexual: atualmente, entende-se que a provável origem dos desfechos de saúde mental citados acima é a vulnerabilidade social gerada por ser parte de um grupo marginalizado (Pachankis et al., 2015). De acordo com o modelo do estresse social, condições sociais podem funcionar como estressores para grupos estigmatizados (Cox, Berghe, Dewaele, \& Vinke, 2008). Supõe-se, portanto, que estressores sociais tenham um impacto forte nas vidas de indivíduos pertencentes a grupos estigmatizados, tais como minorias étnicas, de gênero ou sexuais. $\mathrm{O}$ modelo teórico do estresse de minorias (EM) tem sido indicado como um apanhado explicativo das condições individuais e do meio a ligar a condição de minoria social a desfechos de saúde mental (Meyer, 2015).

A teoria do estresse de minorias (EM) descreve os estressores sociais e individuais específicos de pessoas 
LGB, os quais são distintos dos estressores de outros grupos marginalizados, como pessoas trans e pessoas pertencentes a minorias étnicas. Os três componentes do EM são: experiências de vitimização, homofobia internalizada e ocultação da orientação sexual (Meyer, 2003). Por experiências de vitimização se entende toda discriminação sofrida pelo indivíduo: ou seja, toda fonte externa de homofobia a exercer impacto sobre ele. A vitimização está associada ao desenvolvimento de depressão e estresse pós-traumático em jovens LGB (Mustanski, Andrews, \& Puckett, 2016). Outro componente estressor a ser destacado é a homofobia internalizada (HI), que pode ser entendida como a atitude hostil do próprio indivíduo LGB acerca da sua orientação sexual (Dunn et al., 2013). Assim, a HI é a internalização do estigma referente à orientação sexual pela própria pessoa LGB. Se alguém que é lésbica, gay ou bissexual apresenta atitude negativa frente ao fato de sentir atração física e emocional por pessoas do mesmo gênero, possivelmente as suas crenças a respeito de si serão negativas e distorcidas (Pachankis et al., 2015). A HI pode levar a prejuízos na saúde mental, como o desenvolvimento de transtornos de humor e ansiedade, comportamentos sexuais de risco e abuso de substâncias (Puckett et al., 2017). A ocultação da orientação sexual, por sua vez, está associada a temor de rejeição por parte de pares (Pachankis et al., 2015). Por temer a rejeição dos pares, que estão inseridos em uma sociedade muitas vezes reforçadora do estigma negativo associado a orientações não heterossexuais, as pessoas LGB podem optar por não lhes contar a respeito dessa parte de quem são. Isso pode levá-las a viver em estado de alerta constante, controlando suas condutas e não experienciando uma existência autêntica (Meyer, 2003). A revelação da orientação sexual, por outro lado, parece estar associada a maiores índices de bem-estar e saúde mental (Riggle, Rostosky, Black, \& Rosenkrantz, 2017). Assim, a condição de minoria social faria pessoas LGB experienciarem estressores específicos que, quando associados a fatores de risco e proteção, tais como resiliência, apoio social e conexão com a comunidade LGB, poderiam levar a índices mais altos de bem-estar subjetivo ou psicopatologia (Meyer, 2003).

Porém, a literatura aponta existirem diferenças entre os grupos de gays, lésbicas e bissexuais quanto a seus índices de qualidade de vida e psicopatologia (Shearer et al., 2016). Pessoas bissexuais apresentam níveis mais altos de comportamento suicida, abuso de substâncias e transtornos de depressão e ansiedade quando comparadas a heterossexuais, gays e lésbicas (Pompili et al., 2014). Mulheres lésbicas e bissexuais tendem a apresentar maiores índices suicidas ao longo da vida quando comparadas a mulheres heterossexuais, sendo os índices das mulheres bissexuais os mais altos dentre os três (Shearer et al., 2016). Isso pode acontecer devido a uma maior dificuldade de estabelecer relações de apoio social, já que, além de sofrer estigmatização e discriminação por parte de pessoas heterossexuais, indivíduos bissexuais podem ter dificuldade de formar vínculos com gays e lésbicas (Kertzner, Meyer, \& Frost, 2009). Essa dificuldade é gerada pelos processos de monossexismo e invisibilização da bissexualidade, que produzem um estigma associado especificamente a essa sexualidade, muitas vezes tida como uma fase ou indecisão, além de poder ser entendida como promiscuidade (Flanders, Dobinson, \& Logie, 2015). Assim, muitas vezes, pessoas monossexuais (sejam elas heterossexuais, gays ou lésbicas) acabam por perceber as pessoas bissexuais como não confiáveis, optando por não estabelecer relações com elas (Roberts, Horne, \& Hoyt, 2015). Bissexuais podem, também, internalizar tais estigmas, desenvolvendo crenças negativas a respeito de si e de sua bissexualidade (Pachankis et al., 2015). Como resultado, bissexuais apresentam também maiores índices de confusão identitária e maior ocultação da orientação sexual em comparação a gays e lésbicas (Balsam \& Mohr, 2007), podendo ter o estigma associado à bissexualidade como um obstáculo no caminho de viverem experiências autênticas (Flanders, Dobinson, \& Logie, 2015). Esses processos, quando severos, podem levar a um comprometimento na saúde mental de pessoas desse 
grupo (Pachankis et al., 2015). Por outro lado, a aceitação da bissexualidade por parte de familiares e amigos pode funcionar como um fator preditor de menores níveis de preconceito antibissexual (Roberts, Horne, \& Hoyt, 2015).

Considerando os aspectos acima mencionados, o presente estudo buscou comparar os níveis de bem-estar subjetivo e psicopatologia de mulheres lésbicas, bissexuais e heterossexuais. Como variáveis de bem- estar subjetivo, foram avaliadas felicidade subjetiva e satisfação com a vida, componentes afetivo e cognitivo, respectivamente do conceito de bem-estar (ScorsoliniComin, Fontaine, Barroso, \& dos Santos, 2016). Como variáveis de psicopatologia, foram avaliadas depressão, ansiedade e estresse. A depressão, a ansiedade e o estresse são os quadros psicopatológicos mais recorrentes nessa amostra (Apóstolo, Mendes, \& Azeredo, 2006).

\section{Método}

\section{Participantes}

Participaram deste estudo 736 mulheres, sendo $55,12 \%$ heterossexuais $(n=414), 19,04 \%$ lésbicas $(n=$ $143)$ e $25,83 \%$ bissexuais $(n=194)$, assumidas $(67,96 \%$; $n=227)$ ou não $(32,03 \% ; n=107)$, maiores de 18 anos de idade $(M=27,37 ; D P=13,44)$. Como o processo de revelação da orientação sexual é contínuo, optou-se por perguntar às respondentes se elas se consideravam assumidas ou não, por meio da pergunta "Você se considera assumida para as outras pessoas?" As opções de resposta eram "sim" e "não", uma vez que o objetivo era obter a percepção das respondentes quanto a estarem ou não "no armário". As respondentes em sua maioria viviam em região metropolitana $(83,6 \%)$, com renda familiar de 3 a 10 salários mínimos $(60 \%)$ e ensino superior incompleto $45,7 \%$ ). Foram coletados dados de todas as regiões do País. As participantes, majoritariamente, residiam na região Sudeste, tendo $45,5 \%$ reportado viver no estado do Rio de Janeiro. Não houve cálculo amostral para definição do número de participantes.
Entretanto, com vistas a conduzir adequadamente as análises estatísticas propostas, um número elevado de participantes foi necessário.

\section{Instrumentos}

Questionário sociodemográfico. Este instrumento visa a levantar informações sociodemográficas, com aspectos referentes ao local de residência, ao gênero, à instituição de ensino, à idade, ao estado civil, à constituição familiar, à religião, à escolaridade, ao vínculo empregatício, à renda etc.

Escala de Satisfação com a Vida (Diener et al. 1985, adaptada por Gouveia, Milfont, Fonseca, \& Coelho, 2009). A Escala de Satisfação com a Vida avalia a satisfação do indivíduo a respeito de sua própria vida. Possui cinco itens, que são respondidos em escala Likert de sete pontos, com variação de 1 (discordo totalmente) a 5 (concordo totalmente). No presente estudo, a fidedignidade da escala foi $\alpha=0,89$.

Escala de Felicidade Subjetiva (Damásio, Zanon, \& Koller, 2014). A Escala de Felicidade Subjetiva (EFS) avalia, a partir da percepção das próprias pessoas, os seus níveis de felicidade (componente emocional do bem-estar subjetivo). O instrumento é composto por quatro itens, respondidos em uma escala de sete pontos. No presente estudo, a fidedignidade da escala foi $\alpha=0,89$.

Escala de Depressão, Ansiedade e Stress (DASS -21; Apóstolo, Mendes, \& Azeredo, 2006). O instrumento consiste em 21 itens que investigam indicadores de depressão, ansiedade e estresse, relacionando-a com a qualidade de vida e a personalidade. As respostas podem variar de 0 (não se aplicou a mim de forma nenhuma) a 3 (Aplicou-se muito a mim ou na maior parte do tempo). Neste estudo, a fidedignidade dos fatores foram: Depressão, $\alpha=0,94$; Ansiedade $\alpha=0,89$; Estresse $\alpha=0,91$.

\section{Procedimentos de coleta de dados}

A coleta de dados quantitativa foi realizada por meio de uma plataforma virtual. O link para participação na pesquisa foi divulgado em redes sociais e por e-mail. 
Além disso, foi utilizada a técnica bola de neve, onde participantes do estudo indicam outros possíveis interessados na pesquisa (Patton, 1990).

\section{Procedimentos de análise de dados}

Inicialmente, foram realizadas estatísticas descritivas (média e desvio padrão) de todos os instrumentos para observar a distribuição dos dados na amostra. Posteriormente, foram realizadas correlações de Pearson entre as variáveis sociodemográficas orientação sexual, idade, renda, importância da religião (i. e., quão importante as respondentes consideravam sua religião de escolha) e escolaridade e as variáveis psicológicas (felicidade subjetiva, satisfação com a vida, depressão, ansiedade e estresse). Por fim, buscou-se comparar os níveis de bem-estar (satisfação com a vida e felicidade subjetiva, depressão, estresse e ansiedade) entre mulheres de diferentes orientações sexuais (heterossexuais, lésbicas e bissexuais). Para tanto, foi realizada uma Análise Multivariada de Variância (MANOVA). As MANOVAS foram implementadas com procedimentos de reamostragem (bootstrapping; 1000 reamostragens; intervalo de confiança 99\%). O procedimento de rea- mostragem foi utilizado com vistas a garantir maior confiabilidade aos resultados, além de corrigir possíveis desvios de distribuição de normalidade dos dados, incompatibilidade nos tamanhos dos grupos, além de apresentar um intervalo de confiança de $99 \%$ para as diferenças das médias (Haukoos \& Lewis, 2005).

\section{Procedimentos éticos}

Este estudo foi conduzido após aprovação pelo Comitê de Ética em Pesquisado Centro de Filosofia e Ciências Humanas (CFCH) da Universidade Federal do Rio de Janeiro (UFRJ), sob número de protocolo CAEE: 40922815.5.0000.5582. Todas as suas etapas foram realizadas de maneira ética e metodologicamente adequada, de acordo com as Diretrizes e Normas Regulamentadoras de Pesquisa envolvendo Seres Humanos (Resolução n. 466/12 do Conselho Nacional de Saúde).

\section{Resultados}

A Tabela 1 apresenta as estatísticas descritivas de todas as variáveis mensuradas separadas por grupo a fim de compreender as características da amostra.

\section{Tabela 1.}

Estatísticas descritivas das variáveis de bem-estar mensuradas

\begin{tabular}{|l|l|c|c|c|}
\hline \multicolumn{5}{|c|}{ Estatísticas descritivas } \\
\hline \multirow{5}{*}{ Variável } & \multicolumn{1}{|c|}{ Orientação sexual } & Média & Desvio padrão & 143 \\
\hline \multirow{5}{*}{ Satisfação com a vida } & Lésbicas & 22,81 & 7,79 & 193 \\
\cline { 2 - 5 } & Bissexuais & 21,00 & 7,24 & 398 \\
\cline { 2 - 5 } & Heterossexuais & 23,78 & 6,90 & 734 \\
\cline { 2 - 5 } & Total & 22,86 & 7,26 & 143 \\
\hline \multirow{5}{*}{ Felicidade subjetiva } & Lésbicas & 18,11 & 6,15 & 193 \\
\cline { 2 - 5 } & Bissexuais & 16,41 & 6,17 & 398 \\
\cline { 2 - 5 } & Heterossexuais & 18,57 & 5,96 & 734 \\
\cline { 2 - 5 } & Total & 17,91 & 6,11 & \multirow{2}{*}{} \\
\hline
\end{tabular}




\begin{tabular}{|c|c|c|c|c|}
\hline \multirow{4}{*}{ Depressão } & Lésbicas & 9,49 & 6,04 & 143 \\
\hline & Bissexuais & 11,69 & 5,99 & 193 \\
\hline & Heterossexuais & 7,61 & 5,89 & 398 \\
\hline & Total & 9,04 & 6,19 & 734 \\
\hline \multirow{4}{*}{ Ansiedade } & Lésbicas & 6,95 & 5,06 & 143 \\
\hline & Bissexuais & 8,87 & 5,74 & 193 \\
\hline & Heterossexuais & 5,49 & 4,95 & 398 \\
\hline & Total & 6,66 & 5,38 & 734 \\
\hline \multirow{4}{*}{ Estresse } & Lésbicas & 10,29 & 5,34 & 143 \\
\hline & Bissexuais & 12,02 & 5,38 & 193 \\
\hline & Heterossexuais & 9,26 & 5,20 & 398 \\
\hline & Total & 10,19 & 5,39 & 734 \\
\hline
\end{tabular}

Nota: $N$ - número de respondentes por grupo e amostra total.

A Tabela 2, por sua vez, apresenta as relações biva- escolaridade e importância da religião, pode-se notar riadas entre variáveis sociodemográficas idade, renda, relações estatisticamente significativas positivas com as importância da religião e escolaridade, e indicadores variáveis de bem-estar psicológico. Foram encontradas de bem-estar (felicidade subjetiva, satisfação com a correlações negativas entre as variáveis idade, renda, vida, depressão, ansiedade e estresse) na amostra. Em importância da religião e escolaridade e as variáveis relação às variáveis sociodemográficas idade, renda, depressão, ansiedade e estresse.

\section{Tabela 2.}

Correlações entre as variáveis de bem-estar, psicopatologia e indicadores sociodemográficos

\begin{tabular}{|c|c|c|c|c|c|c|c|c|c|}
\hline & Idade & Renda & $\begin{array}{l}\text { Imp. da } \\
\text { religião }\end{array}$ & Escolaridade & Depressão & Ansiedade & Estresse & $\begin{array}{c}\text { Felicidade } \\
\text { subjetiva }\end{array}$ & $\begin{array}{c}\text { Satis. com } \\
\text { a vida }\end{array}$ \\
\hline Idade & - & & & & & & & & \\
\hline Renda & 0,066 & - & & & & & & & \\
\hline $\begin{array}{l}\text { Imp. da } \\
\text { religião }\end{array}$ & $0,174^{* *}$ & $-0,084^{*}$ & - & & & & & & \\
\hline Escolaridade & $0,274^{* *}$ & $0,184^{* *}$ & $0,138^{* *}$ & - & & & & & \\
\hline Depressão & $-0,182^{* *}$ & $-0,095^{*}$ & $-0,172^{* *}$ & $-0,254^{* *}$ & - & & & & \\
\hline Ansiedade & $-0,162^{* *}$ & $-0,090^{*}$ & $-0,114^{* *}$ & $-0,256^{* *}$ & $0,737^{* *}$ & - & & & \\
\hline Estresse & $-0,139^{* *}$ & $-0,077^{*}$ & $-0,123^{* *}$ & $-0,194^{* *}$ & $0,752^{* *}$ & $0,804^{* *}$ & - & & \\
\hline
\end{tabular}




\begin{tabular}{|c|c|c|c|c|c|c|c|c|c|}
\hline $\begin{array}{c}\text { Felicidade } \\
\text { subjetiva }\end{array}$ & $0,134^{* *}$ & $0,089^{*}$ & $0,243^{* *}$ & $0,179^{* *}$ & $-0,633^{* *}$ & $-0,397^{* *}$ & $-0,422^{* *}$ & - \\
\hline $\begin{array}{c}\text { Satis. com a } \\
\text { vida }\end{array}$ & $0,072^{*}$ & $0,219^{* *}$ & $0,152^{* *}$ & $0,199^{* *}$ & $-0,539^{* *}$ & $-0,329^{* *}$ & $-0,356^{* *}$ & $0,691^{* *}$ & - \\
\hline
\end{tabular}

Nota: ** $p<0,01 ; * p<0,05$; Imp. da religião - importância da religião; Satis. Com a vida - satisfação com a vida

Devido à relação estatisticamente significativa entre renda, escolaridade e importância da religião nas variáveis psicológicas depressão, ansiedade, estresse, felicidade subjetiva e satisfação com a vida, optou-se por inseri-las como variáveis de controle na análise múltipla de variância, sendo executada, portanto, uma MANCOVA, que teve por objetivo comparar os níveis de bem-estar e psicopatologia em mulheres com diferentes orientações sexuais (heterossexual, homossexual e bissexual).

As covariáveis renda, escolaridade e importância da religião apresentaram impacto significativo nos re- sultados [Renda familiar, $F(723,5)=7,446, p<0,00$; Escolaridade, $F(723,5)=5,693, p<0,01$; Importância da religião, $F(723,5)=7,345, p<0,01]$. A idade, por sua vez, não apresentou impacto significativo (Idade, $F(723$, $5)=2,076, p=0,66)$. Os resultados da MANCOVA demonstraram que a orientação sexual apresentou impacto significativo em todos os indicadores de bem-estar avaliados (ver Tabela 3), com tamanho de efeito baixo para as variáveis satisfação com a vida, felicidade subjetiva e estresse e moderado para depressão e ansiedade.

\section{Tabela 3.}

Resultados da Análise Multivariada de Variância (MANOVA) entre orientação sexual e indicadores de bem-estar

\begin{tabular}{|l|c|c|c|c|}
\hline \multicolumn{1}{|c|}{ Variável dependente } & F & gl & Sig & Tamanho de Efeito $\left(\boldsymbol{\eta}^{2}\right)$ \\
\hline Satisfação com a vida & 4,353 & $(2,731)$ & 0,013 & 0,012 \\
\hline Felicidade subjetiva & 3,242 & $(2,731)$ & 0,040 & 0,009 \\
\hline Depressão & 17,767 & $(2,731)$ & 0,000 & 0,047 \\
\hline Ansiedade & 16,563 & $(2,731)$ & 0,000 & 0,044 \\
\hline Estresse & 10,266 & $(2,731)$ & 0,000 & 0,027 \\
\hline
\end{tabular}

Foram executadas análises post-hoc a posteriori (Bonferroni), com vistas a identificar quais grupos se diferenciavam entre si nas variáveis analisadas (ver Tabela 4). No que se refere à satisfação com a vida, mulheres bissexuais apresentaram menores índices de satisfação com a vida quando comparadas a mulheres heterossexuais. Não houve diferença estatisticamente significativa nos índices de satisfação com a vida entre mulheres lésbicas em comparação com mulheres bissexuais. Para felicidade subjetiva, por sua vez, embora os resultados gerais da MANCOVA tenham apontado para diferenças estatis- ticamente significativas entre mulheres heterossexuais e não heterossexuais, as análises post-hoc demonstraram que os efeitos eram apenas marginalmente significativos.

No que diz respeito aos índices de depressão, ansiedade e estresse, mulheres bissexuais tiveram escores significativamente maiores quando comparado com os grupos de hetero e homossexuais. As mulheres lésbicas, por sua vez, tiveram escores equivalentes aos das mulheres heterossexuais (ver Tabela 4; dados descritivos na Tabela 1). 


\section{Tabela 4.}

Análises post-hoc para avaliação de diferenças de médias de BES e PSP entre grupos

\begin{tabular}{|c|c|c|c|c|c|c|}
\hline \multirow{2}{*}{ Variável dependente } & \multirow{2}{*}{\multicolumn{2}{|c|}{$\begin{array}{c}\text { Orientação sexual } \\
\text { (Grupos de comparação) }\end{array}$}} & \multirow{3}{*}{$\begin{array}{c}\Delta M \\
1,541\end{array}$} & \multirow{3}{*}{$\begin{array}{c}\text { Sig } \\
\text { Limite } \\
\text { inferior }\end{array}$} & \multicolumn{2}{|c|}{$\begin{array}{c}\text { Intervalo de confiança } \\
\qquad(95 \%)\end{array}$} \\
\hline & & & & & Limite & \\
\hline \multirow{3}{*}{ Satisfação com a vida } & \multirow{2}{*}{ Lésbicas } & Bissexuais & & & $-0,297$ & 3,380 \\
\hline & & Hetero & $-0,272$ & 1,000 & $-1,913$ & 1,370 \\
\hline & Hetero & Bissexuais & $1,813^{*}$ & 0,012 & 0,312 & 3,314 \\
\hline \multirow{3}{*}{ Felicidade subjetiva } & \multirow{2}{*}{ Lésbicas } & Bissexuais & 1,448 & 0,078 & $-0,108$ & 3,003 \\
\hline & & Hetero & 0,270 & 1,000 & $-1,119$ & 1,659 \\
\hline & Hetero & Bissexuais & 1,177 & 0,079 & $-0,093$ & 2,448 \\
\hline \multirow{3}{*}{ Depressão } & \multirow{2}{*}{ Lésbicas } & Bissexuais & $-1,795^{*}$ & 0,016 & $-3,333$ & $-0,257$ \\
\hline & & Hetero & 1,317 & 0,065 & $-0,056$ & 2,691 \\
\hline & Hetero & Bissexuais & $-3,112^{*}$ & 0,000 & $-4,368$ & $-1,856$ \\
\hline \multirow{3}{*}{ Ansiedade } & \multirow{2}{*}{ Lésbicas } & Bissexuais & $-1,575^{*}$ & 0,016 & $-2,925$ & $-0,225$ \\
\hline & & Hetero & 1,065 & 0,103 & $-0,140$ & 2,271 \\
\hline & Hetero & Bissexuais & $-2,640^{*}$ & 0,000 & $-3,743$ & $-1,538$ \\
\hline \multirow{3}{*}{ Estresse } & \multirow{2}{*}{ Lésbicas } & Bissexuais & $-1,456^{*}$ & 0,035 & $-2,840$ & $-0,072$ \\
\hline & & Hetero & 0,676 & 0,568 & $-0,559$ & 1,912 \\
\hline & Hetero & Bissexuais & $-2,132^{*}$ & 0,000 & $-3,262$ & $-1,003$ \\
\hline
\end{tabular}

Nota: sig - significância estatística; $\Delta M=$ diferença de médias.

\section{Discussão}

No presente estudo, foram encontradas relações significativas entre as variáveis sociodemográficas testadas e as variáveis de bem-estar e psicopatologia selecionadas. As variáveis renda, escolaridade, importância da religião e idade se relacionaram positivamente com felicidade subjetiva e satisfação com a vida, e negativamente com os índices de depressão, ansiedade e estresse. Neste sentido, compreende-se que quanto maiores a renda, a escolaridade, a importância da religião praticada e a idade, maiores os níveis de felicidade subjetiva e de satisfação com a vida nas participantes, e menores os seus índices de depressão, ansiedade e estresse. Tais resultados encontram apoio em achados de outros estudos. A renda está associada a maior satisfação com a vida (Kahneman \& Deaton, 2010), enquanto a ausência de renda ou estágios mais graves de pobreza parece ter relação com desfechos negativos de saúde mental, como a depressão (Mendenhall, Kohrt, Norris, Ndetei, \& Prabhakaran, 2017). A escolaridade também tem relação com os desfechos de saúde mental, podendo desempenhar um papel protetivo contra o desenvolvimento de ansiedade e depressão (Bjelland et al., 2008; 
Lozupone et al., 2017). A religiosidade tende a ser um fator protetivo importante, auxiliando a manutenção de componentes do bem-estar como satisfação com a vida e sentido de vida (Tanaka, Ho, \& Johnson, 2017). Em pessoas LGB, a idade pode funcionar como fator protetivo em decorrência de sua condição de minoria: como o processo de revelação da orientação sexual tende a ocorrer durante a juventude, entende-se que ao longo do tempo as pessoas pertencentes a esse grupo tendem a desenvolver estratégias de enfrentamento e redes de apoio social que permitam o aumento de seu bem-estar (Meyer, 2003; Pachankis et al., 2015). As correlações, embora significativas, foram baixas. Esses resultados estão de acordo com a literatura, uma vez que de acordo com o modelo do Estresse de Minorias (EM), as condições sociodemográficas podem impactar os níveis dos estressores de minorias de pessoas LGB (Meyer, 2003). Como o modelo do EM apresenta diversas relações entre múltiplas variáveis, faz sentido que os tamanhos de efeito das relações citadas sejam baixos: muitas outras variáveis (identidade de minoria, estressores gerais, características da identidade de minoria, estratégias de enfrentamento, entre outras) também podem impactar os índices de EM e saúde mental de pessoas LGB (Pachankis et al., 2015).

O EM postula que crenças negativas sobre si ou sobre a própria sexualidade, assim como atos de violência derivados da condição de minoria, colocam indivíduos LGB em posição vulnerável em relação a pessoas heterossexuais (Meyer, 2003; Stuber, Meyer, \& Link, 2008). As análises estatísticas sugerem que mulheres lésbicas e bissexuais apresentam menores níveis de bem-estar (felicidade subjetiva e satisfação com a vida) e maiores níveis de psicopatologia (depressão, estresse e ansiedade) do que mulheres heterossexuais. Acredita-se que esses indicadores estão relacionados ao EM. Ou seja, supõe-se que por fazer parte da população LGB, as mulheres não heterossexuais apresentaram níveis mais altos de depressão e ansiedade do que as heterossexuais na presente amostra. Os tamanhos de efeito apresentados na
Tabela 3 demonstraram que os resultados encontrados (ou seja, $4,4 \%$ dos escores de ansiedade e $4,7 \%$ dos escores de depressão apresentados pela população de lésbicas e bissexuais) podem ser explicados apenas pelo fato de fazerem parte de um grupo estigmatizado. Ser parte de um grupo estigmatizado significa estar exposto a ações discriminatórias e a processos de estigmatização que mantêm o indivíduo em constante estado de alerta e impactam o desenvolvimento de crenças negativas distorcidas a respeito de si (Stuber, Meyer, \& Link, 2008). Mulheres lésbicas e bissexuais podem ter a sua vulnerabilidade aumentada em decorrência de se manterem em alerta, à espera de alguma ação violenta discriminatória, constantemente (Dunn et al., 2013). Além disso, o processo de estigmatização afeta o desenvolvimento daqueles que fazem parte destes grupos, pois influencia no desenvolvimento de crenças disfuncionais a respeito de si e de sua sexualidade (Pachankis et al., 2015).

As análises post-hoc mostraram um maior impacto negativo na saúde mental de mulheres bissexuais do que na de mulheres lésbicas e heterossexuais, onde as primeiras apresentaram níveis mais baixos de satisfação com a vida e felicidade subjetiva e maiores escores de depressão, ansiedade e estresse quando comparadas com seus pares heterossexuais. Mulheres bissexuais também apresentaram níveis mais baixos de felicidade subjetiva e mais altos de depressão, ansiedade e estresse quando comparadas a mulheres lésbicas. Supõe-se que isto se deva ao que se entende por bifobia, um tipo de discriminação específico relacionado à população bissexual, que pode vir tanto de heterossexuais quanto da população de gays e lésbicas através dos processos de invisibilização e deslegitimização da bissexualidade como expressão possível da sexualidade (Wandrey, Mosack, \& Moore, 2015). Assim, devido ao processo de invisibilização, a bissexualidade muitas vezes é entendida como um período de indecisão ou um processo de mudança da heterossexualidade para a homossexualidade: por isso, pessoas bissexuais podem ser discriminadas por pessoas 
heterossexuais e pela comunidade de gays e lésbicas (Kertzner, Meyer, \& Frost, 2009).

A bifobia pode impactar as crenças a respeito da bissexualidade (como "bissexualidade é uma fase" e "pessoas bissexuais são promíscuas", por exemplo), podem levar a um cenário excludente para aqueles que integram este grupo, dificultando a formação de vínculos sociais com pessoas que sentem atração por apenas um gênero, como heterossexuais, gays e lésbicas (Pachankis et al., 2015). Tais crenças podem, também, impactar o autoconceito de pessoas bissexuais, ampliando os seus índices de bifobia internalizada e os sentimentos negativos frente a si. Considerando a interseccionalidade do modelo do EM, compreende-se que quanto mais status de minoria uma pessoa possuir, mais estressores ela terá, e consequentemente mais afetada será sua saúde mental (Meyer, 2003). Pessoas bissexuais possuem os estressores comuns a pessoas não heterossexuais, como homofobia internalizada, experiências de vitimização e ocultação da orientação sexual, mas além disso também podem apresentar bifobia internalizada e discriminação específica relacionada à bissexualidade (Wandrey, Mosack, \& Moore, 2015).

Além disso, existe também um estigma de gênero em relação à identidade bissexual de mulheres: por vezes, considera-se que pessoas do gênero feminino têm uma fase bissexual para a apreciação masculina (Wandrey, Mosack, \& Moore, 2015). Isto é, supõe-se que mulheres podem emitir comportamentos sexuais entre si a fim de entreter seus parceiros do sexo oposto, sugerindo a bissexualidade feminina como uma fase voltada para o agrado masculino. Conforme a literatura indica, é possível que esse tipo de discriminação afete os índices de apoio social de indivíduos bissexuais e, somada à bifobia internalizada, aumente as chances de desenvolvimento de ansiedade, depressão e estresse (Kertzner, Meyer, \& Frost, 2009). Resultados similares quanto aos níveis de saúde mental e bem-estar foram encontrados no presente estudo, o que pode sugerir padrões de sociabilização similares aos de estudos internacionais para os grupos de indivíduos bissexuais respondentes.

Não houve diferença estatisticamente significativa nos índices de satisfação com a vida entre mulheres lésbicas em comparação com mulheres bissexuais. A satisfação com a vida é o componente cognitivo do bem-estar subjetivo: nesse sentido, o conceito faz referência a uma avaliação cognitiva da pessoa a respeito de sua própria vida e de em que medida ela satisfaz as suas expectativas prévias (Diener, Emmons, Larsen, \& Griffin, 1985). Em pessoas LGB, a autoestima e a solidão estão, respectivamente, positiva e negativamente associadas à satisfação com a vida (Hu, Hu, Huang, \& Zheng, 2016). Mesmo que pessoas bissexuais possam apresentar maiores níveis de EM e estressores específicos associados à bissexualidade, na presente amostra não houve diferença entre mulheres lésbicas e bissexuais quanto à sua avaliação cognitiva a respeito da própria vida.

Os baixos tamanhos de efeito das diferenças entre os índices de saúde mental da amostra podem ser justificados tendo em vista que a orientação sexual de uma pessoa não é o único fator determinante em suas relações sociais e, portanto, a vulnerabilidade social gerada pelo status de minoria não exclui os estressores gerais com os quais todos têm contato (Dunn et al., 2013). Desse modo, é possível que haja vulnerabilidade social na amostra em decorrência de sua posição de minoria social, e que essa vulnerabilidade social tenha impactado os níveis de felicidade subjetiva, satisfação com a vida, depressão, ansiedade e estresse das mulheres bissexuais e lésbicas que responderam à pesquisa. Porém, talvez neste grupo de respondentes, tal diferença não seja tão impactante. Isso pode se dever ao fato de a amostra ser bastante homogênea: em sua maioria, mulheres jovens $(M=27,37 ; D P=13,44)$, residentes de região metropolitana (83,6\%), com renda familiar de 3 a 10 salários mínimos (60\%) e Ensino Superior Incompleto 45,7\%). Assim, as condições de vida das participantes podem ser consideradas similares, tornando as suas experiências de vida também homogêneas. Por isso, as 
baixas diferenças entre os grupos se justificam: sendo a orientação sexual apenas uma característica dentre muitas que são parte da realidade humana, e a única (dentre as variáveis analisadas) que dá às respondentes experiências de vida diferentes, devido ao estigma associado às orientações não heterossexuais.

\section{Considerações finais}

De acordo com a teoria do Estresse de Minorias (EM), pessoas não heterossexuais, por serem parte de uma minoria social, apresentam vulnerabilidade social e maiores índices de psicopatologias como depressão e ansiedade do que seus pares heterossexuais (Meyer, 2003). O objetivo do presente estudo foi comparar os níveis de felicidade subjetiva, satisfação com a vida, depressão, ansiedade e estresse entre mulheres heterossexuais, lésbicas e bissexuais brasileiras. Os índices de depressão, ansiedade e estresse mostraram-se mais altos para a população de lésbicas e bissexuais quando comparadas a mulheres heterossexuais. Os índices de felicidade subjetiva e satisfação com a vida reportados foram mais baixos para as mulheres não heterossexuais, quando comparadas às heterossexuais. A amostra de mulheres bissexuais, especificamente, apresentou menores índices de felicidade subjetiva e satisfação com a vida e maiores níveis de depressão, ansiedade e estresse do que seus pares lésbicas e heterossexuais. Embora os dados estejam de acordo com a literatura, os tamanhos de efeito das diferenças entre os grupos foram baixos. Isto pode ter acontecido devido a uma limitação do estudo: como a estratégia para a divulgação da pesquisa online foi a técnica da bola de neve (Patton, 1990), a amostra respondente foi bastante homogênea, o que pode ter sido refletido nos tamanhos de efeito baixos. Considerando esse panorama, compreende-se a importância do presente estudo para a divulgação da realidade de mulheres e de minorias sexuais no que diz respeito à saúde mental no País. Os dados coletados são úteis na medida em que funcionam como um levanta- mento de bem-estar e saúde mental da população LGB feminina quando comparada a seus pares heterossexuais, auxiliando na construção de assistência mais adequada e promoção de políticas públicas em prol dessa comunidade. Como o objetivo do presente estudo foi comparar os níveis de indicadores de bem-estar e psicopatologia em mulheres heterossexuais, lésbicas e bissexuais, não se pôde fazer inferências referentes aos níveis de EM da amostra. Pesquisas mais amplas, com amostras mais heterogêneas, buscando compreender melhor os efeitos de processos de estigmatização e discriminação na saúde mental e no bem-estar da população LGB e buscando investigar possíveis moderadores dessa relação, devem ser feitas, a fim de promover melhorias mais efetivas no fazer da psicologia destinada ao grupo.

\section{Referências}

Apóstolo, J. L. A., Mendes, A. C., \& Azeredo, Z. A. (2006). Adaptação para a língua portuguesa da Depression, Anxiety and Stress Scale (DASS). Revista Latino-americana de Enfermagem, 14(6). doi: 10.1590/ S0104-11692006000600006

Balsam, K. F., \& Mohr, J. J. (2007). Adaptation to sexual orientation stigma: A comparison of bisexual and lesbian/gay adults. Journal of Counseling Psychology, 54(3), 306-319. doi: 10.1037/0022-0167.54.3.306

Bjelland, I., Krokstad, S., Mykletun, A., Dahl, A. A., Tell, G. S., \& Tambs, K. (2008). Does a higher educational level protect against anxiety and depression? The HUNT study. Social science \& medicine, 66(6), 1334-1345. doi: 10.1016/j.socscimed.2007.12.019

Borrillo, D. (2010). Homofobia: história e crítica de um preconceito. Belo Horizonte, Brasil: Autêntica Editora.

Brown, T. A. (2006). Confirmatory factor analysis for applied research. Nova York, EUA: The Guilford Press.

Cox, N., Van den Berghe, W., Dewaele, A., \& Vincke, J. (2008). General and minority stress in an LGB population in Flanders. Journal of LGBT Health Research, 4(4), 181-194. doi:10.1080/15574090802657168

Damásio, B. F., Zanon, C., \& Koller, S. H. (2014). Validation and psychometric properties of the Brazilian 
version of the Subjective Happiness Scale. Universitas Psychologica, 13(1), 1-13. doi: 10.11144/Javeriana. UPSY13-1.vppb

Diener, E. D., Emmons, R. A., Larsen, R. J., \& Griffin, S. (1985). The satisfaction with life scale. Journal of personality assessment, 49(1), 71-75. doi: 10.1207/ s15327752jpa4901_13

Donahue, K., Långström, N., Lundström, S., Lichtenstein, P., \& Forsman, M. (2017). Familial Factors, Victimization, and Psychological Health Among Sexual Minority Adolescents in Sweden. American Journal of Public Health, (0), el-e7. doi: 10.2105/AJPH.2016.303573

Dunn, T. L., Gonzalez, C. A., Costa, A. B., Nardi, H. C., \& Iantaffi, A. (2014). Does the Minority Stress Model Generalize to a Non-US Sample? An Examination of Minority Stress and Resilience on Depressive Symptomatology Among Sexual Minority Men in Two Urban Areas of Brazil. Psychology of Sexual Orientation and Gender Diversity, 1(2), 117-131. doi: $10.1037 /$ sgd0000032

Flanders, C. E., Dobinson, C., \& Logie, C. (2015). “I'm Never Really My Full Self”: Young Bisexual Women's Perceptions of their Mental Health. Journal of Bisexuality, 15(4), 454-480. doi: 10.1080/15299716.2015.1079288

Gouveia, V. V., Milfont, T. L., Fonseca, P. N., \& Coelho, J. A. P. M. (2008). Life Satisfaction in Brazil: Testing the Psychometric Properties of the Satisfaction With Life Scale (SWLS) in Five Brazilian Samples. Social Indicators Research, 90(1), 267-277. doi: 10.1007/ s11205-008-9257-0

Haukoos, J. S., \& Lewis, R. J. (2005). Advanced statistics: Bootstrapping confidence intervals for statistics with "difficult" distributions. Academic Emergency Medicine, 12(4), 360-365. doi:10.1197/j.aem.2004.11.018

Horn, J. L. (1965).A rationale and technique for estimating the number of factors in factor analysis. Psychometrika, 30(1), 179-185. doi: 10.1007/BF02289447

Hu, J., Hu, J., Huang, G., \& Zheng, X. (2016). Life satisfaction, self-esteem, and loneliness among LGB adults and heterosexual adults in China. Journal of homosexuality, 63(1), 72-86. doi: 10.1080/00918369.2015.1078651

Kahneman, D., \& Deaton, A. (2010). High income improves evaluation of life but not emotional well- -being. Proceedings of the national academy of sciences, 107(38), 16489-16493. doi: 10.1073/pnas.1011492107

Kertzner, R. M., Meyer, I. H., Frost, D. M., \& Stirratt, M. J. (2009). Social and psychological well-being in lesbians, gay men, and bisexuals: the effects of race, gender, age, and sexual identity. American Journal of Orthopsychiatry, 79(4), 500. doi: 10.1037/a0016848

Lorenzo-Seva, U., Timmerman, M. E., \& Kiers, H. A. (2011). The Hull method for selecting the number of common factors. Multivariate Behavioral Research, 46(2), 340-364. doi: 10.1080/00273171.2011.564527

Lozupone, M., Veneziani, F., Lofano, L., Galizia, I., Stella, E., Copetti, M., ... Tursi, M. (2017). Educational level influenced the gold standard diagnosis of late-life depression in the GreatAGE study. European Psychiatry, 41, S173. doi: 10.1016/j.eurpsy.2017.01.2068 Mayock, P., Bryan, A., Carr, N., \& Kitching, K. (2008). Supporting LGBT lives: a study of mental health and well-being. Dublin, Irlanda: BeLonG To Youth Services.

Mendenhall, E., Kohrt, B. A., Norris, S. A., Ndetei, D., $\&$ Prabhakaran, D. (2017). Non-communicable disease syndemics: poverty, depression, and diabetes among low-income populations. The Lancet, 389(10072), 951963. doi: 10.1016/S0140-6736(17)30402-6

Meyer, I. H. (2003). Prejudice, Social Stress, and Mental Health in Lesbian, Gay, and Bisexual Populations: Conceptual Issues and Research Evidence. Psychological Bulletin, 129(5), 674-697. doi: 10.1037/00332909.129.5.674

Meyer, I. H. (2015). Resilience in the study of minority stress and health of sexual and gender minorities. Psychology of Sexual Orientation and Gender Diversity, 2(3), 209 -213. doi: 10.1037/sgd0000132

Mustanski, B., Andrews, R., \& Puckett, J. A. (2016). The effects of cumulative victimization on mental health among lesbian, gay, bisexual, and transgender adolescents and young adults. American Journal of Public Health, 106(3), 527-533. doi: 10.2105/AJPH. 2015.302976

Muthén, L K, \& Muthén, B. O. (2010). Mplus: Statistical analysis with latent variables. User's guide. Los Angeles, EUA: Muthén \& Muthén. https://doi. org $/ 10.2307 / 2286403$

Pachankis, J. E., Hatzenbuehler, M. L., Rendina, H. J., Safren, S. A., \& Parsons, J. T. (2015). LGB-affirmative 
cognitive-behavioral therapy for young adult gay and bisexual men: A randomized controlled trial of a transdiagnostic minority stress approach. Journal of Consulting and Clinical Psychology, 83(5), 875-889. doi: $10.1037 / \mathrm{ccp} 0000037$

Patton, M. Q. (1990). Qualitative Evaluation and Research Methods. Newbury Park, EUA: Sage Publications, Inc.

Pompili, M., Lester, D., Forte, A., Seretti, M. E., Erbuto, D., Lamis, D. A., ... Girardi, P. (2014). Bisexuality and suicide: a systematic review of the current literature. The Journal of Sexual Medicine, 11(8), 1903-1913. doi: $10.1111 /$ jsm. 12581

Puckett, J. A., Newcomb, M. E., Ryan, D. T., Swann, G., Garofalo, R., \& Mustanski, B. (2017). Internalized homophobia and perceived stigma: A validation study of stigma measures in a sample of young men who have sex with men. Sexuality Research and Social Policy, 14(1), 1-16. doi: 10.1007/s13178-016-0258-5

Riggle, E. D., Rostosky, S. S., Black, W. W., \& Rosenkrantz, D. E. (2017). Outness, concealment, and authenticity: Associations with LGB individuals' psychological distress and well-being. Psychology of Sexual Orientation and Gender Diversity, 4(1), 54-62. doi: $10.1037 /$ sgd0000202

Roberts, T. S., Horne, S. G., \& Hoyt, W. T. (2015). Between a gay and a straight place: Bisexual individuals' experiences with monosexism. Journal of Bisexuality, 15(4), 554-569. doi: 10.1080/15299716.2015.1111183

Russell, S. T., Ryan, C., Toomey, R. B, Diaz, R. M., \& Sanchez J. (2011). Lesbian, gay, bisexual and transgender adolescent school victimization: implications for young adult health and adjustment. Journal of School Health, 81(5), 223-230. doi: 10.1111/j.1746-1561.2011.00583.x

Ryan, C., Huebner, D., Diaz, R. M., \& Sanchez J. (2009). Family rejection as a predictor of negative health outcomes in white and Latino lesbian, gay, and bisexual young adults. Pediatrics, 123(1), 346-352 doi: 10.1542/ peds.2007-3524

Schmitt, M. T., Branscombe, N. R., Kobrynowicz, D., \& Owen, S. (2002). Perceiving discrimination against one's gender group has different implications for well-being in women and men. Personality and Social Psychology Bulletin, 28(2), 197-210. doi: 10.1177/0146167202282006
Scorsolini-Comin, F., Fontaine, A. M. G. V., Barroso, S. M., \& dos Santos, M. A. (2016). Fatores associados ao bem-estar subjetivo em pessoas casadas e solteiras. Estudos de Psicologia, 33(2), 313-324. Retirado de https://www.redalyc.org/pdf/3953/395354131013.pdf, em 18 de dezembro de 2018. https://doi.org/10.1590/1982$\underline{02752016000200013}$

Shearer, A., Herres, J., Kodish, T., Squitieri, H., James, K., Russon, J., ... Diamond, G. S. (2016). Differences in mental health symptoms across lesbian, gay, bisexual, and questioning youth in primary care settings. Journal of Adolescent Health, 59(1), 38-43. doi: 10.1016/j.jadohealth.2016.02.005

Tanaka, K., Ho, J. H., \& Johnson, N. E. (2017). Linking Religiosity to Life Satisfaction in Japan and the United States. Yearbook of International Religious Demography 2017, 122. https://doi.org/10.1163/9789004346307006

Wandrey, R. L.; Mosack, K. E.; \& Moore, E. M. (2015). Coming Out to Family and Friends as Bisexually Identified Young Adult Women: A Discussion of Homophobia, Biphobia, and Heteronormativity. Journal of Bisexuality, 15(2), 204-229. doi: 10.1080/15299716. 2015.1018657

Endereço para correspondência: Fernanda de Oliveira Paveltchuk (Av. Pasteur, 250 - Urca, Rio de Janeiro - RJ, 22290-240; (21) 97474-3158; paveltchuk@ gmail.com).982638203; quadroscherer@gmail.com).

Recebido em: 7/8/2018.

Aprovado em: 16/1/2019.

Publicado em: xx/xx/2019. 